\title{
Fluid-solid mixtures and electrochemomechanics: the simplicity of Lagrangian mixture theory
}

\author{
JACQUES M. HUYGHE, R. VAN LOON and F.T.P. BAAIJENS \\ Department of Biomedical Engineering, Eindhoven University of Technology \\ Eindhoven, The Netherlands \\ E-mail: j.m.r.huyghe@tue.nl
}

\begin{abstract}
Today, the focus of physical scientists is shifting more to biology than ever before. A biological tissue is typically an ionised porous medium saturated with a solution of ions and neutral solutes. Because classical porous media theories do not account for ionisation, the present paper addresses this issue. The characteristic pore size in most biological applications is close to the molecular level and hence below the Debye-Hueckel scale. Not only pressure gradients and concentration gradients, but electrical gradients as well are intimately linked to fluid flow, ion flow and deformation.
\end{abstract}

Mathematical subject classification: 76ZXX, 76W05, 76S05.

Key words: swelling, shale, cartilage, finite deformation, equipresence.

\section{Introduction}

Since antiquity, the phenomenon of swelling of tissues has been closely related to health and disease. Biological, synthetic and mineral porous media often exhibit swelling or shrinking when in contact with changing salt concentrations. This phenomenon, observed in clays, shales, cartilage and gels, is caused by a combination of electrostatic forces and hydration forces [11]. In case of biological tissue, electrostatic forces are often dominant. Classical concepts, such as the transmembrane potential of cells are directly associated with these electrostatic forces. Already years ago, Biot understood that his theories were closely associated with transmembrane phenomena in living cells [4]. At least four \#573/03. Received: 14/IV/03. Accepted: 29/IV/04. 
components are involved in the swelling mechanics: a solid, a fluid, anions and cations. Lai et al. [11] developed a triphasic theory for soft hydrated tissue and applied the theory to cartilage while neglecting geometric non-linearities. They verified the theory for one-dimensional equilibrium results. As soft tissues and cells are commonly subject to large deformations, our group developed a finite deformation theory of ionised media [9]. In order to simplify the mathemetics as much as possible a Lagrangian form of the entropy inequality has been derived which leads to equations consistent with Biot's porous media theories in a more straightforward way than the more familiar Eulerian approach of Bowen [6]. The incompressibility and electroneutrality conditions are introduced by means of two Lagrange multipliers; the latter is physically interpreted as an electrical potential, the former as a pressure.

\section{Fluid-solid mixtures}

We shall derive equations applicable to the behaviour of elastic incompressible fluid saturated porous media from mixture theory.

\subsection{Assumptions}

We consider the porous medium as a two-component mixture, composed of a solid (superscript $s$ ) and a fluid component (superscript $f$ ). Saturation requires:

$$
\varphi^{s}+\varphi^{f}=1 .
$$

We assume that no mass-exchange occurs between the components. Each component is assumed incompressible:

$$
\rho_{i}^{\alpha}=\frac{\rho^{\alpha}}{\varphi^{\alpha}}=\text { constant }, \quad \alpha=s, f .
$$

The apparent densities $\rho^{\alpha}$ however do change as a function of time. We consider processes which are sufficiently slow so as to ensure that inertia forces are negligible. Volume forces are neglected as well. We assume that all components have the same temperature and no gradients in temperature are present either in time or space. 


\subsection{Conservation laws}

\subsubsection{Conservation of mass}

In the absence of mass exchange the local law of conservation of mass of component $\alpha$ reduces to:

$$
\frac{\partial \rho^{\alpha}}{\partial t}+\nabla \cdot\left(\rho^{\alpha} \boldsymbol{v}^{\alpha}\right)=0, \quad \alpha=s, f
$$

We can rewrite (3):

$$
\frac{\partial \varphi^{\alpha}}{\partial t}+\nabla \cdot\left(\varphi^{\alpha} \boldsymbol{v}^{\alpha}\right)=0, \quad \alpha=s, f
$$

Summation of the equations (4) yields the local mass balance of the mixture:

$$
\nabla \cdot\left(\varphi^{s} \boldsymbol{v}^{s}\right)+\nabla \cdot\left(\varphi^{f} \boldsymbol{v}^{f}\right)=0
$$

or:

$$
\boldsymbol{\nabla} \cdot \boldsymbol{v}^{s}+\nabla \cdot\left(\varphi^{f}\left(\boldsymbol{v}^{f}-\boldsymbol{v}^{s}\right)\right)=0 .
$$

The first term of (6) represents the rate of volume increase of a unit volume of mixture. The second term represents the fluid flux from this unit volume. Eq. (6) states that every volume-increase or decrease of the mixture is associated with an equal amount of in- or outflux of liquid. At this point it is useful to refer current descriptors of the mixture with respect to an initial state of the porous solid. As is usual in continuum mechanics, we define the deformation gradient tensor $\boldsymbol{F}$ mapping an infinitesimal material line segment in the initial state onto the corresponding infinitesimal line segment in the current state. The relative volume change from the initial to the current state is the determinant of the deformation gradient tensor $J=\operatorname{det} \boldsymbol{F}$. If we introduce volume fractions

$$
\Phi^{\alpha}=J \varphi^{\alpha}
$$

per unit initial volume, we can rewrite the mass balance equation (4) as follows:

$$
\frac{D^{s} \Phi^{\alpha}}{D t}+J \nabla \cdot\left[\varphi^{\alpha}\left(\boldsymbol{v}^{\alpha}-\boldsymbol{v}^{s}\right)\right]=0
$$

when using the identity:

$$
\frac{D^{s}}{D t} J=J \nabla \cdot \boldsymbol{v}^{s}
$$




\subsubsection{Conservation of momentum}

Considering the assumptions stated earlier, momentum balance reduces to:

$$
\boldsymbol{\nabla} \cdot\left(\boldsymbol{\sigma}^{\alpha}\right)^{c}+\hat{\boldsymbol{p}}^{\alpha}=\mathbf{0}, \quad \alpha=s, f .
$$

The momentum interaction $\hat{\boldsymbol{p}}^{\alpha}$ arises e.g., as a consequence of friction between the fluid and the solid. We assume no moment of momentum interaction between fluid and solid. Therefore we tacitly assumed the symmetry of the partial Cauchy stress tensor in (10). Summation of the equations (10) yields the local momentum balance for the mixture as a whole:

$$
\boldsymbol{\nabla} \cdot \boldsymbol{\sigma}^{s}+\nabla \cdot \boldsymbol{\sigma}^{f}=\boldsymbol{\nabla} \cdot \boldsymbol{\sigma}=\mathbf{0},
$$

if we use:

$$
\hat{\boldsymbol{p}}^{s}+\hat{\boldsymbol{p}}^{f}=\mathbf{0}
$$

\subsubsection{The entropy inequality}

The local form of the entropy inequality applied to the mixture as a whole, reduces to:

$$
\sum_{\alpha=s, f}\left(-\rho^{\alpha} \frac{D^{\alpha} \tilde{F}^{\alpha}}{D t} \tilde{F}^{\alpha}+\boldsymbol{\sigma}^{\alpha}: \boldsymbol{D}^{\alpha}-\hat{\boldsymbol{p}}^{\alpha} \cdot \boldsymbol{u}^{\alpha}\right) \geq 0 .
$$

We introduce the strain energy function

$$
W=J \sum_{\alpha=s, f} \rho^{\alpha} \tilde{F}^{\alpha}=J \sum_{\alpha=s, f} \psi^{\alpha}
$$

as the Helmholtz free energy of a mixture volume which in the initial state of the solid equals unity. $\psi^{\alpha}$ is the Helmholz free energy of constituent $\alpha$ per unit mixture volume. Rewriting the inequality (13) for the entropy production per initial mixture volume - i.e. we multiply inequality (13) by the relative volume change $J$ - we find:

$$
-\frac{D^{s}}{D t} W+J \boldsymbol{\sigma}: \nabla \boldsymbol{v}^{s}+J \nabla \cdot\left[\left(\boldsymbol{v}^{f}-\boldsymbol{v}^{s}\right) \cdot \boldsymbol{\sigma}^{f}-\left(\boldsymbol{v}^{f}-\boldsymbol{v}^{s}\right) \psi^{f}\right] \geq 0 .
$$




\subsection{Constitutive restrictions}

We use the entropy inequality to derive constitutive restrictions for the mixture. The entropy inequality should hold for an arbitrary state of the mixture, complying with the balance laws and with incompressibility. There are two ways to comply with these restrictions. One is substitution of the restriction into the inequality, resulting in elimination of a field variable. The other is by introduction of a Lagrange multiplier. The mass balance of the mixture (6) is accounted for by means of a Lagrange multiplier. Other balance laws and the incompressibility conditions (2) are accounted for by means of substitution. From the inequality 15 we see that the apparent density and the momentum interaction $\hat{\boldsymbol{p}}^{\alpha}$ is already eliminated from the inequality. In other words the conditions of incompressibility and the momentum balance of the constituents have already been substituted into the second law. The divergence of the partial stress tensor of the solid $\boldsymbol{\nabla} \cdot \boldsymbol{\sigma}^{s}$ and the heat supplies $r^{\alpha}$ also are absent from 15. Thus the momentum balance of the mixture and the energy balance have already been substituted in the second law. Therefore, restrictions still to be fulfilled are the mass balances of the constituents (3) and mass balance of the mixture (6). The latter is substituted by means of a Lagrange multiplier $p$ :

$$
\begin{aligned}
-\frac{D^{s}}{D t} W & +J \boldsymbol{\sigma}_{e}: \boldsymbol{\nabla} \boldsymbol{v}^{s}+J\left[\boldsymbol{\sigma}^{f}+\left(p \varphi^{f}-\psi^{f}\right) \boldsymbol{I}\right]: \boldsymbol{\nabla}\left(\boldsymbol{v}^{f}-\boldsymbol{v}^{s}\right) \\
& +J\left(\boldsymbol{v}^{f}-\boldsymbol{v}^{s}\right) \cdot\left(-\nabla \psi^{f}+p \boldsymbol{\nabla} \varphi^{f}+\boldsymbol{\nabla} \cdot \boldsymbol{\sigma}^{f}\right) \geq 0 .
\end{aligned}
$$

in which the effective stress $\sigma_{e}$ is defined as

$$
\sigma_{e}=\sigma+p \boldsymbol{I}
$$

\subsubsection{Choice of independent and dependent variables}

We choose as dependent variables the dynamic variables appearing in inequality 16: $W, \psi^{f}, \boldsymbol{\sigma}_{e}, \boldsymbol{\sigma}^{f}+p \varphi^{f} \boldsymbol{I}, \boldsymbol{\nabla} \cdot \boldsymbol{\sigma}^{f}+p \boldsymbol{\nabla} \varphi^{f}$. Their number should equate the number unknown variables appearing in the balance equations minus the number of balance equations. The number of dependent variables should be as small as possible to describe the state of the tissue well. Their choice is a key assumption of the continuum theory and is based on insight in the physical 
phenomena involved in the behaviour of the material. We choose as independent variables the kinematic variables: the Green strain of the solid $\boldsymbol{E}^{s}$, the fluid volume fraction $\Phi^{f}$ and the fluid velocity relative to the solid $\boldsymbol{v}^{f}-\boldsymbol{v}^{s}$. For reasons of objectivity we need to transform all the vectors and tensors among the dependent and independent variables back to the initial state. This yields for the constitutive relationships:

$$
\begin{aligned}
W & =W\left(\boldsymbol{E}^{s}, \Phi^{f}, \boldsymbol{v}^{f s}\right), \\
\psi^{f} & =\psi^{f}\left(\boldsymbol{E}^{s}, \Phi^{f}, \boldsymbol{v}^{f s}\right), \\
\boldsymbol{\sigma}_{e} & =\boldsymbol{F} \cdot \boldsymbol{S}_{e}\left(\boldsymbol{E}^{s}, \Phi^{f}, \boldsymbol{v}^{f s}\right) \cdot \boldsymbol{F}^{c}, \\
\boldsymbol{\sigma}^{f}-\varphi^{f} p \boldsymbol{I} & =\boldsymbol{F} \cdot \boldsymbol{S}^{f}\left(\boldsymbol{E}^{s}, \Phi^{f}, \boldsymbol{v}^{f s}\right) \cdot \boldsymbol{F}^{c} \\
\hat{\boldsymbol{p}}^{f}-p \boldsymbol{\nabla} \varphi^{f} & =\boldsymbol{F} \cdot \hat{\boldsymbol{P}}^{f}\left(\boldsymbol{E}^{s}, \Phi^{f}, \boldsymbol{v}^{f s}\right)
\end{aligned}
$$

with

$$
\boldsymbol{v}^{f s}=\boldsymbol{F}^{-1} \cdot\left(\boldsymbol{v}^{f}-\boldsymbol{v}^{s}\right)
$$

The principle of equipresence requires that all dependent variables appear in each of the constitutive relationships. The choice of the independent variables is paramount for the form of the constitutive relationships that are derived. E.g., including for the solid Green strain only and no measure of strain rate, implies elasticity of the solid. In mixture mechanics it is also important to realise that each of the variables is an averaged value of a physical quantity over an averaging volume. It may seem surprising that the shear rate of the fluid is not included in the list of independent variables, although the viscosity of the fluid is absolutely essential for the behaviour of the mixture. The reason for this is that in a porous medium the shear rate at one side of the pore has a sign opposite to the shear rate at the other side of the pore. The expectation value of the shear rate in a representative elementary volume is therefore the shear rate of the solid, i.e. a generally very low value, not representative for the dissipation in the fluid. It is therefore more obvious to use the fluid velocity relative to the solid as a macroscopic measure of the microvalues of the shear rate. The fluid volume fraction $\Phi^{f}$ is not independent of the Green strain because of incompressibility:

$$
\Phi^{f}=\operatorname{det} \boldsymbol{F}-1+\varphi_{0}^{f}=\sqrt{\operatorname{det}\left(2 \boldsymbol{E}^{s}+\boldsymbol{I}\right)}-1+\varphi_{0}^{f}
$$


Because of the strong non-linearity of equation (20), elimination of one of the variables is tedious. In fact, the way we deal with the interdependence of these two variables is by means of the Lagrange multiplier p. The condition (6) is in fact a differentated form of equation (20). This legitimises the use of $\boldsymbol{E}^{s}$ and $\Phi^{f}$ as independent variables.

\subsubsection{Constitutive relationships}

Applying the chain rule for time differentiation of $\mathrm{W}$ :

$$
\frac{D^{s} W}{D t}=\frac{\partial W}{\partial \boldsymbol{E}^{s}}: \frac{D^{s} \boldsymbol{E}^{s}}{D t}+\frac{\partial W}{\partial \Phi^{f}} \frac{D^{s} \Phi^{f}}{D t}+\frac{\partial W}{\partial \boldsymbol{v}^{f s}}
$$

and substituting the mass balance of the constituents (8) for the elimination of $\frac{D^{s} \Phi^{f}}{D t}$ from the inequality 16 :

$$
\begin{aligned}
\left(J \boldsymbol{\sigma}_{e}\right. & \left.-\boldsymbol{F} \cdot \frac{\partial W}{\partial \boldsymbol{E}} \cdot \boldsymbol{F}^{c}\right): \boldsymbol{\nabla} \boldsymbol{v}^{s}+\frac{\partial W}{\partial \boldsymbol{v}^{f s}} \cdot \frac{D^{s}}{D t} \boldsymbol{v}^{f s} \\
& +J\left[\boldsymbol{\sigma}^{f}+\left(\mu^{f} \varphi^{f}-\psi^{f}\right) \boldsymbol{I}\right]: \boldsymbol{\nabla}\left(\boldsymbol{v}^{f}-\boldsymbol{v}^{s}\right) \\
& +J\left(\boldsymbol{v}^{f}-\boldsymbol{v}^{s}\right) \cdot\left(-\boldsymbol{\nabla} \psi^{f}+\mu^{f} \boldsymbol{\nabla} \varphi^{f}+\boldsymbol{\nabla} \cdot \boldsymbol{\sigma}^{f}\right) \geq 0 .
\end{aligned}
$$

in which $\mu^{f}$ is the chemical potential of the fluid:

$$
\mu^{f}=\frac{\partial W}{\partial \Phi^{f}}+p
$$

Eq. (22) should be true for any value of the state variables. Close inspection of the choice of independent variables and the inequality (22), reveals that the first term of (22) is linear in the solid velocity gradient $\boldsymbol{\nabla} \boldsymbol{v}^{s}$, the second term linear in $\frac{D^{s}}{D t} \boldsymbol{v}^{f s}$ and the third term linear in the relative velocity gradients $\boldsymbol{\nabla}\left(\boldsymbol{v}^{f}-\boldsymbol{v}^{s}\right)$. Therefore, by a standard argument, we find:

$$
\begin{aligned}
& \boldsymbol{\sigma}_{e}=\frac{1}{J} \boldsymbol{F} \cdot \frac{\partial W}{\partial \boldsymbol{E}} \cdot \boldsymbol{F}^{c} \\
& \frac{\partial W}{\partial \boldsymbol{v}^{f s}}=\mathbf{0} \\
& \boldsymbol{\sigma}^{f}=\left(\psi^{f}-\mu^{f} \varphi^{f}\right) \boldsymbol{I}
\end{aligned}
$$


leaving as inequality:

$$
J\left(\boldsymbol{v}^{f}-\boldsymbol{v}^{s}\right) \cdot\left(-\boldsymbol{\nabla} \psi^{f}+\mu^{f} \nabla \varphi^{f}+\boldsymbol{\nabla} \cdot \boldsymbol{\sigma}^{f}\right) \geq 0 .
$$

Eq. (24) indicates that the effective stress of the mixture can be derived from a strain energy function $\mathrm{W}$ which represents the free energy of the mixture. Eq. (25) shows that the strain energy function cannot depend on the relative velocity of fluid versus solid. This result - only obtained in a Lagrangian formulation - simplifies the constitutive laws to a large extent, because a vectorial variable disappears among the independent variables of the free energy $W$, and its derivatives, the effective stress and the chemical potential. The partial free energies, $\psi^{s}$ and $\psi^{f}$ cannot be shown independent from the relative velocity. Thus, the effective stress of a biphasic medium can be derived from a regular strain energy function, which physically has the same meaning as in single phase media. According to equation (26) the partial stress of the fluid is a scalar. Transforming the relative velocities to their Lagrangian equivalents, we find in stead of (27):

$$
\boldsymbol{v}^{f s} \cdot\left[-\nabla_{0} \psi^{f}+\mu^{f} \nabla_{0} \varphi^{f}+\nabla_{0} \cdot \boldsymbol{\sigma}^{f}\right] \geq 0 .
$$

in which $\nabla_{0}=\boldsymbol{F}^{c} \cdot \nabla$ is the gradient operator with respect to the initial configuration. Note that as $\mu^{f} \boldsymbol{\nabla}_{0} \varphi^{f}+\nabla_{0} \cdot \boldsymbol{\sigma}^{f}$ depends on $\boldsymbol{v}^{f s}$ according to the constituive relationships (18), the lefthandside of inequality (28) is not a linear function of $\boldsymbol{v}^{f s}$ and therefore it is incorrect to equate the factor $-\boldsymbol{\nabla}_{0} \psi^{f}+\mu^{f} \boldsymbol{\nabla}_{0} \varphi^{f}+\boldsymbol{\nabla}_{0} \cdot \boldsymbol{\sigma}^{f}$ to zero. From a physical point of view it is obvious that unlike the elastic deformation of the solid the flow of fluid relative the solid results in an entropy production. If we assume that the system is not too far from equilibrium, we can express the dissipation (28) associated with relative flow of fluid and ions as a quadratic function of the relative velocities:

$$
-\nabla_{0} \psi^{f}+\mu^{f} \nabla_{0} \varphi^{f}+\nabla_{0} \cdot \boldsymbol{\sigma}^{f}=\boldsymbol{B} \cdot \boldsymbol{v}^{f s}
$$

$\boldsymbol{B}$ is a semi-positive definite matrix of frictional coefficients. Substituting equation (26) into equation (29) yields the Lagrangian form of Darcy's law:

$$
-\varphi^{f} \nabla_{0} \mu^{f}=\boldsymbol{B} \cdot \boldsymbol{v}^{f s}
$$

The constitutive behaviour of the fluid-solid mixture is thus described by a strain energy function $W$ and frictional tensor $\boldsymbol{B}$. From the strain energy function we derive both the effective stress and the chemical potential of the fluid. 


\subsection{Physical interpretation of the constitutive variables}

The Lagrange multiplier $\mathrm{p}$ should be interpreted as the hydrostatic pressure in the fluid.

$$
\boldsymbol{\nabla} \cdot \boldsymbol{\sigma}_{e}-\nabla p=\mathbf{0}
$$

If we define the permeability tensor $\boldsymbol{K}$ as:

$$
\boldsymbol{K}=\left(\varphi^{f}\right)^{2} \boldsymbol{B}^{-1}
$$

equation (30) becomes:

$$
\varphi^{f}\left(\boldsymbol{v}^{f}-\boldsymbol{v}^{s}\right)=-\boldsymbol{K} \cdot \nabla\left(p+\frac{\partial W}{\partial \Phi^{f}}\right) .
$$

Eq. (33) is the threedimensional form of Darcy's law. The difference between the chemical potential $\mu^{f}$ and the pressure $p$ is the matric potential. The matric potential accounts for adsorption and capillary forces. It can be quantified experimentally using capillary rising heights. In Terzaghi's consolidation theory the matric potentisal is neglected, not because it is negligible in absolute terms but because its gradient is negligible in an homogenous medium with limited variation of fluid volume fraction and coarse pore structure.

\subsection{Resulting equations}

The resulting equations are:

Momentum balance of the mixture:

$$
\boldsymbol{\nabla} \cdot \boldsymbol{\sigma}_{e}-\nabla p=\mathbf{0}
$$

Mass balance of the mixture:

$$
\boldsymbol{\nabla} \cdot \boldsymbol{v}^{s}-\boldsymbol{\nabla} \cdot\left(\varphi^{f}\left(\boldsymbol{v}^{f}-\boldsymbol{v}^{s}\right)\right)=0
$$

Darcy's law:

$$
\boldsymbol{v}^{f}-\boldsymbol{v}^{s}=\varphi^{f} \boldsymbol{B}^{-1} \cdot \nabla \mu^{f}
$$


Stress-strain relationship:

$$
\boldsymbol{\sigma}_{e}=(\operatorname{det} \boldsymbol{F})^{-1} \boldsymbol{F} \cdot \frac{\partial W}{\partial \boldsymbol{E}^{s}} \cdot \boldsymbol{F}^{c},
$$

Constitutive law for the chemical potential of the fluid:

$$
\mu^{f}=p+\frac{\partial W}{\partial \Phi^{f}}
$$

The total stress in the mixture is composed of an effective stress and a hydrodynamic pressure: $\boldsymbol{\sigma}=\boldsymbol{\sigma}_{e}-p \boldsymbol{I}$. The effective stress $\boldsymbol{\sigma}_{e}$ is derived from the strain energy function of the mixture $W$. In equation (38) $\boldsymbol{F}$ is the deformation gradient tensor of the solid and $\boldsymbol{E}^{s}$ the Green strain tensor of the solid. The strain energy $W$ in a function of the solid strain $\boldsymbol{E}$.

Dynamic boundary conditions are:

$$
\left[\left(\boldsymbol{\sigma}_{e}-p \boldsymbol{I}\right) \cdot \boldsymbol{n}\right]=\mathbf{0}
$$

with $\boldsymbol{n}$ the outer normal along the boundary and the square brackets represent the difference between the value at either side of the boundary.

$$
\left[\bar{V}^{f} \mu^{f}\right]=0
$$

with as a special case the evaporation boundary condition:

$$
\bar{V}^{f} \mu^{f}=R T \ln \frac{p^{d}}{p_{s}^{d}}
$$

Equation (40) and (41) enforces continuity of molar chemical potential. Discontinuity of chemical potential would lead to an infinite fluid flux which is physically impossible. For this reason we can claim that even if the material properties are sharply discontinuous the chemical potential should be continuous. This is not true for the pressure p. E.g. at the interface between a sand layer and a clay layer the pressure is not continuous, at least if capillary effects are not neglected. Similarly interstititial pressure along the surface of the skin is not equal to atmospheric pressure. We use the molar chemical potential in stead of the volumetric chemical potential because the volumetric chemical potential can be used only for incompressible media. Although the medium we consider 
is incompressible, the medium outside the boundary need not be incompressible as is the case for evaporation. Kinematic boundary conditions are:

$$
\begin{aligned}
& {[\boldsymbol{u}]=\mathbf{0}} \\
& {\left[\left(\boldsymbol{v}^{f}-\boldsymbol{v}^{s}\right) \cdot \boldsymbol{n}\right]=0}
\end{aligned}
$$

\section{Donnan Osmosis}

When an ionised medium is in contact with a monovalent salt solution, diffusion of salt ions and flow of fluid take place between the medium and the salt solution until equilibrium is reached:

$$
\begin{aligned}
& \mu^{+}=\bar{\mu}^{+} \\
& \mu^{-}=\bar{\mu}^{-} \\
& \mu^{f}=\bar{\mu}^{f}
\end{aligned}
$$

$\mu^{+}$is the electrochemical potential of the cations, $\mu^{-}$is the electrochemical potential of the anions and $\mu^{f}$ the chemical potential of the fluid in the medium. The corresponding overlined symbols refer to chemical potentials in the outer solution. Standard expressions for (electro)chemical potentials are found in the literature [15]. If we assume incompressibility for each constituent, i.e. same partial molar volumes in either solution, we find:

$$
\begin{aligned}
& \mu^{+}=\mu_{0}^{+}+\frac{1}{\bar{V}^{+}}\left(R T \ln a^{+}+F \xi\right) \\
& \mu^{-}=\mu_{0}^{-}+\frac{1}{\bar{V}^{-}}\left(R T \ln a^{-}-F \xi\right) \\
& \mu^{f}=\mu_{0}^{f}+p+\frac{R T}{\bar{V}^{f}} \ln a^{f}
\end{aligned}
$$

in which $\mu_{0}^{\beta}$ are reference values, $\bar{V}^{\beta}$ partial molar volumes, $a^{\beta}$ activities, $p$ the fluid pressure, $T$ absolute temperature, $R$ universal gas constant, $F$ Faraday's constant and $\xi$ the electrical potential. All of these (electro)chemical potentials are measured here per unit volume constituent. Combination of equation (44) 
and (45) leads to:

$$
\begin{aligned}
& a^{-} a^{+}=\bar{a}^{+} \bar{a}^{-} \\
& \xi-\bar{\xi}=\frac{R T}{2 F} \ln \frac{a^{-} \bar{a}^{+}}{a^{+} \bar{a}^{-}}
\end{aligned}
$$

where $\xi-\bar{\xi}$ is the Donnan potential between the inner and outer solution. If we define $c^{f c}$ as the fixed charge density per unit fluid volume of the inner solution, taken positive for positive charges and negative for negative charges, we can write the electroneutrality conditions as:

$$
\begin{aligned}
& c^{-}=c^{+}+c^{f c} \\
& \bar{c}^{-}=\bar{c}^{+}=\bar{c}
\end{aligned}
$$

$c^{+}$and $c^{-}$are the cationic and anionic concentrations per unit fluid volume in the inner solution, while the corresponding overlined symbols pertain to the outer solution. In terms of the volume fractions introduced in equation (7), the concentrations are

$$
c^{\beta}=\frac{\Phi^{\beta}}{\Phi^{f} \bar{V}^{\beta}}
$$

From the previous equations we derive the Donnan equilibrium concentration of the ions:

$$
\begin{aligned}
& 2 c^{+}=-c^{f c}+\sqrt{\left(c^{f c}\right)^{2}+4 f^{2} \bar{c}^{2}} \\
& 2 c^{-}=c^{f c}+\sqrt{\left(c^{f c}\right)^{2}+4 f^{2} \bar{c}^{2}}
\end{aligned}
$$

with

$$
f^{2}=\frac{\bar{f}^{+} \bar{f}^{-}}{f^{+} f^{-}}
$$

and $f^{\beta}=\frac{a^{\beta}}{c^{\beta}}, \quad \beta=+,-$ the activity coefficient of component $\beta$. Equations (55-56) show that the cationic concentration jumps to a higher and the anionic concentration to a lower value when entering the porous medium. These concentration jumps are responsible for the attraction of water into the porous medium 
during swelling and for the associated osmotic pressure $\pi$. Using equation (49) one can derive Van't Hoff relation from (46):

$$
\pi=p-\bar{p}=R T\left[\Gamma^{f}\left(c^{+}+c^{-}\right)-2 \bar{\Gamma}^{f} \bar{c}\right]
$$

provided that the molar fractions of the ions are small compared to the molar fraction of the fluid. $\Gamma^{f}$ and $\bar{\Gamma}^{f}$ are the osmotic coefficients.

\section{Quadriphasic theory}

It may be clear from the above considerations that physical phenomena occuring in the porous medium are a combination of mechanical, chemical and electrical effects. The interrelationship between these effects are well known for membrane processes [16]. The purpose of this paper is to generalise these relationships for porous media subjected to threedimensional finite deformation. The four phases that we consider in the medium are: solid (superscript s), fluid (superscript $\mathrm{f}$ ), monovalent anions (superscript - ) and monovalent cations (superscript + ). Assuming all components intrinsically incompressible and excluding mass transfer between phases, the mass balance of each phase is given by (4), where $\alpha$ takes the values $\mathrm{s}, \mathrm{f},+$ or - . We assume saturation

$$
\varphi^{s}+\varphi^{f}+\varphi^{+}+\varphi^{-}=1 .
$$

Summation of the equation (4) yields the mass balance of the mixture:

$$
\vec{\nabla} \cdot \vec{v}^{s}+\sum_{f,+,-} \vec{\nabla} \cdot\left(\varphi^{\alpha}\left(\vec{v}^{\alpha}-\vec{v}^{s}\right)\right)=0
$$

The electrostatic interactions are accounted for by means of an electroneutrality condition:

$$
\frac{D^{s}}{D t} \sum_{\beta=f,+,-} \frac{z^{\beta} \Phi^{\beta}}{\bar{V}^{\beta}}=0
$$

We introduce the strain energy function as the Helmholtz free energy of a mixture volume which in the initial state of the solid equals unity. $\psi^{\alpha}$ is the 


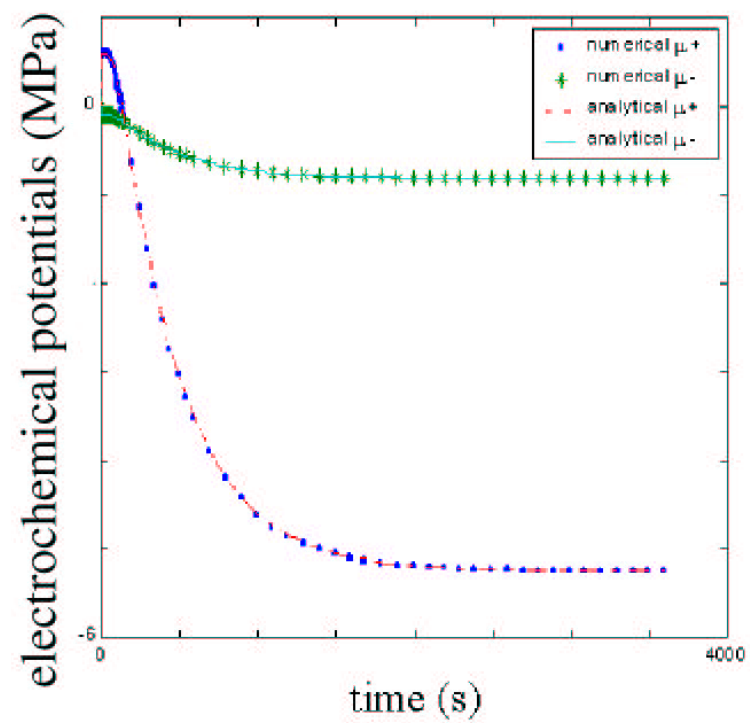

Figure 1 - Electrochemical potential of anions and cations as a function of time during swelling of a one dimensional ionised medium. The solution from a 3D finite element code [17] is compared to the analytical solution [18].

Helmholz free energy of constituent $\alpha$ per unit mixture volume. The inequality for the entropy production per initial mixture volume reads:

$$
\begin{gathered}
-\frac{D^{s}}{D t} W+J \sigma: \vec{\nabla} \vec{v}^{s} \\
+J \vec{\nabla} \cdot \sum_{\beta=f,+,-}\left[\left(\vec{v}^{\beta}-\vec{v}^{s}\right) \cdot \sigma^{\beta}-\left(\vec{v}^{\beta}-\vec{v}^{s}\right) \psi^{\beta}\right] \geq 0 .
\end{gathered}
$$

We assume the existence of a Lagrange multiplier $p$ for the saturation condition and $\lambda$ for the electroneutrality condition. The entropy inequality transforms into:

$$
\begin{gathered}
-\frac{D^{s}}{D t} W+J \boldsymbol{\sigma}^{e f f}: \vec{\nabla} \vec{v}^{s} \\
+J \sum_{\beta=f,+,-}\left[\boldsymbol{\sigma}^{\beta}+\left(\left(p+\frac{z^{\beta} \lambda}{\bar{V}^{\beta}}\right) \varphi^{\beta}-\psi^{\beta}\right) \boldsymbol{I}\right]: \vec{\nabla}\left(\vec{v}^{\beta}-\vec{v}^{s}\right) \\
+J \sum_{\beta=f,+,-}\left(\vec{v}^{\beta}-\vec{v}^{s}\right) \cdot\left[-\vec{\nabla} \psi^{\beta}+\left(p+\frac{z^{\beta} \lambda}{\bar{V}^{\beta}}\right) \vec{\nabla} \varphi^{\beta}+\vec{\nabla} \cdot \boldsymbol{\sigma}^{\beta}\right] \geq 0 .
\end{gathered}
$$


in which $z^{\beta}$ is the valence of constituent $\beta, \sigma^{e f f}$ is the effective stress of the medium. We choose as independent variables the Green strain $\boldsymbol{E}$, the Lagrangian form of the volume fractions of the fluid and the ions $\Phi^{\beta}$, and of the relative velocities $\vec{v}^{\beta s}=\boldsymbol{F}^{-1} \cdot\left(\vec{v}^{\beta}-\vec{v}^{s}\right), \beta=f,+,-$. We apply the principle of equipresence asd the chain rule for time differentiation of $W$ :

$$
\begin{gathered}
\left(J \boldsymbol{\sigma}^{e f f}-\boldsymbol{F} \cdot \frac{\partial W}{\partial \boldsymbol{E}} \cdot \boldsymbol{F}^{c}\right): \vec{\nabla} \vec{v}^{s} \\
+\sum_{\beta=f,+,-}\left\{\frac{\partial W}{\partial \vec{v}^{\beta s}} \cdot \frac{D^{s}}{D t} \vec{v}^{\beta s}+J\left[\boldsymbol{\sigma}^{\beta}+\left(\mu^{\beta} \varphi^{\beta}-\psi^{\beta}\right) \boldsymbol{I}\right]: \vec{\nabla}\left(\vec{v}^{\beta}-\vec{v}^{s}\right)\right. \\
\left.+J\left(\vec{v}^{\beta}-\vec{v}^{s}\right) \cdot\left(-\vec{\nabla} \psi^{\beta}+\mu^{\beta} \vec{\nabla} \varphi^{\beta}+\vec{\nabla} \cdot \boldsymbol{\sigma}^{\beta}\right)\right\} \geq 0 .
\end{gathered}
$$

in which $\mu^{\beta}$ are the electrochemical potentials of fluid and ions:

$$
\begin{aligned}
& \mu^{f}=\frac{\partial W}{\partial \Phi^{f}}+p \\
& \mu^{+}=\frac{\partial W}{\partial \Phi^{+}}+\frac{\lambda}{\bar{V}^{+}}+p \\
& \mu^{-}=\frac{\partial W}{\partial \Phi^{-}}-\frac{\lambda}{\bar{V}^{-}}+p
\end{aligned}
$$

By a standard argument [7], we find:

$$
\begin{aligned}
& \boldsymbol{\sigma}^{e f f}=\frac{1}{J} \boldsymbol{F} \cdot \frac{\partial W}{\partial \boldsymbol{E}} \cdot \boldsymbol{F}^{c} \\
& \frac{\partial W}{\partial \vec{v}^{\beta s}}=\mathbf{0} \\
& \boldsymbol{\sigma}^{\beta}=\left(\psi^{\beta}-\mu^{\beta} \varphi^{\beta}\right) \boldsymbol{I}
\end{aligned}
$$

leaving as inequality:

$$
\sum_{\beta=f,+,-} J\left(\vec{v}^{\beta}-\vec{v}^{s}\right) \cdot\left(-\vec{\nabla} \psi^{\beta}+\mu^{\beta} \vec{\nabla} \varphi^{\beta}+\vec{\nabla} \cdot \sigma^{\beta}\right) \geq 0 .
$$

Equation (66) indicates that the effective stress of the mixture can be derived from a strain energy function $\mathrm{W}$ which represents the free energy of the mixture. 
Equation (67) shows that, again, the strain energy function cannot depend on the relative velocities. In fact this result can easily be generalised to mixtures of arbitrary number of components. Thus, the effective stress of a quadriphasic medium can be derived from a regular strain energy function, which physically has the same meaning as in single phase or biphasic media, but which can depend on both strain and ion concentrations in the medium. According to equation (68) the partial stress of the fluid and the ions are scalars. Transforming the relative velocities to their Lagrangian equivalents, we find in stead of (69):

$$
\sum_{\beta=f,+,-} \vec{v}^{\beta s} \cdot\left[-\vec{\nabla}_{0} \psi^{\beta}+\mu^{\beta} \vec{\nabla}_{0} \varphi^{\beta}+\vec{\nabla}_{0} \cdot \sigma^{\beta}\right] \geq 0 .
$$

in which $\vec{\nabla}_{0}=\boldsymbol{F}^{c} \cdot \vec{\nabla}$ is the gradient operator with respect to the initial configuration. If we assume that the system is not too far from equilibrium, we can express the dissipation (70) associated with relative flow of fluid and ions as a quadratic function of the relative velocities:

$$
-\vec{\nabla}_{0} \psi^{\beta}+\mu^{\beta} \vec{\nabla}_{0} \varphi^{\beta}+\vec{\nabla}_{0} \cdot \boldsymbol{\sigma}^{\beta}=\sum_{\gamma=f,+,-} \boldsymbol{B}^{\beta \gamma} \cdot \vec{v}^{\gamma s}
$$

$\boldsymbol{B}^{\beta \gamma}$ is a positive definite matrix of frictional tensors. Substituting equation (68) into equation (71) yields Lagrangian forms of the classical equations of irreversible thermodynamics:

$$
-\varphi^{\beta} \vec{\nabla}_{0} \mu^{\beta}=\sum_{\gamma=f,+,-} \boldsymbol{B}^{\beta \gamma} \cdot \vec{v}^{\gamma s}
$$

The momentum balance equation (11), the mass balance equation (60), the frictional equations (72), the constitutive relationships for the electrochemical potentials (65) and of the effective stress (66) form a set of partial differential equations. The boundary conditions are given by a no-jump condition of the electrochemical potential of the ions and the fluid across the boundary and the momentum balance of the boundary. In our application we choose the mixing 
part of the energy function as

$$
\begin{gathered}
W\left(\Phi^{f}, \Phi^{+}, \Phi^{-}\right)=\mu_{0}^{f} \Phi^{f}+\mu_{0}^{+} \Phi^{+}+\mu_{0}^{-} \Phi^{-} \\
-R T \Gamma\left(\frac{\Phi^{+}}{\bar{V}^{+}}+\frac{\Phi^{-}}{\bar{V}^{-}}\right) \ln \left(\Phi^{f}\right)+R T \frac{\Phi^{+}}{\bar{V}^{+}}\left(\ln \left(\frac{\Phi^{+}}{\bar{V}^{+}}\right)-1\right) \\
+R T \frac{\Phi^{-}}{\bar{V}^{-}}\left(\ln \left(\frac{\Phi^{-}}{\bar{V}^{-}}\right)-1\right)
\end{gathered}
$$

Hence the expressions 65, take the form:

$$
\begin{aligned}
& \mu^{f}=-R T \Gamma\left(\frac{\Phi^{+}}{\Phi^{f} \bar{V}^{+}}+\frac{\Phi^{-}}{\Phi^{f} \bar{V}^{-}}\right)+p \\
& \mu^{+}=R T \ln \frac{\Phi^{+}}{\left(\Phi^{f}\right)^{\Gamma} \bar{V}^{+}}+\frac{\lambda}{\bar{V}^{+}}+p \\
& \mu^{-}=R T \ln \frac{\Phi^{-}}{\left(\Phi^{f}\right)^{\Gamma} \bar{V}^{-}}-\frac{\lambda}{\bar{V}^{-}}+p
\end{aligned}
$$

which is consistent with the classical expressions for electrochemical potentials (47-49), provided that we identify the activity of ion $\beta$ as

$$
a^{\beta}=f^{\beta} c^{\beta}=\frac{\Phi^{\beta}}{\left(\Phi^{f}\right)^{\Gamma} \bar{V}^{\beta}}
$$

the activity coefficient as

$$
f^{\beta}=\left(\Phi^{\beta}\right)^{1-\Gamma}
$$

and the Lagrange multiplier $\lambda$ as the product of the electrical potential and Faraday constant:

$$
\lambda=F \xi
$$

Equations (74-76) justifies the form of the mixing energy (73) as the mixing energy belonging to the Donnan osmosis model. Rearranging equation (65) yields,

$$
\mu^{\alpha}-p-\frac{z^{\alpha}}{\bar{V}^{\alpha}} \lambda=\sum_{\beta=f,+,-} \boldsymbol{C}_{\alpha \beta}^{-1} \Phi^{\beta}
$$


in which,

$$
\begin{aligned}
\boldsymbol{C}_{\alpha \beta}= & {\left[\frac{\partial^{2} W\left(\Phi^{f}, \Phi^{+}, \Phi^{-}\right)}{\partial \Phi^{\alpha} \partial \Phi^{\beta}}\right]^{-1} } \\
= & {\left[\begin{array}{ccc}
R T \Gamma\left(\frac{\Phi^{+}}{\bar{V}^{+}\left(\Phi^{f}\right)^{2}}+\frac{\Phi^{-}}{\bar{V}^{-}\left(\Phi^{f}\right)^{2}}\right) & -\frac{R T \Gamma}{\bar{V}^{+} \Phi^{f}} & -\frac{R T \Gamma}{\bar{V}^{-} \Phi^{f}} \\
-\frac{R T \Gamma}{\bar{V}^{+} \Phi^{f}} & \frac{R T}{\bar{V}^{+} \Phi^{+}} & 0 \\
-\frac{R T \Gamma}{\bar{V}^{-} \Phi^{f}} & 0 & \frac{R T}{\bar{V}^{-} \Phi^{-}}
\end{array}\right]^{-1} }
\end{aligned}
$$

is the inverse of the Hessian of the mixing energy. To obtain the weak formulation the equations are multiplied by arbitrary, time independent weighing functions and integrated over the volume of the mixture $(\Omega)$. The momentum equation is multiplied by a weighing function $\vec{w}_{x}$. The saturation condition, mass equation and equation for electroneutrality are multiplied by the weighing functions $w_{p}$, $w_{\mu}^{\alpha}$ and $w_{\xi}$, respectively. After partial integration and applying the divergence theorem, we find,

$$
\left\{\begin{array}{l}
\int_{\Omega}\left(\vec{\nabla} \vec{w}_{x}\right)^{c}: \sigma d \Omega=\int_{\Gamma} \vec{w}_{x} \cdot(\sigma \cdot \vec{n}) d \Gamma \\
\int_{\Omega} w_{p}\left(\vec{\nabla} \cdot \vec{v}^{s}\right) d \Omega-\int_{\Omega} w_{p} \frac{1}{J} \sum_{\beta} \frac{D^{s} \Phi^{\beta}}{D t} d \Omega=0 \\
\int_{\Omega} w_{\mu}^{\alpha} \frac{1}{J} \frac{D^{s} \Phi^{\beta}}{D t} d \Omega+\int_{\Omega}\left(\sum_{\alpha} \boldsymbol{K}_{\alpha \beta} \vec{\nabla} \mu^{\alpha}\right) \cdot \vec{\nabla} w_{\mu}^{\alpha} d \Omega \\
=\int_{\Gamma} w_{\mu}^{\alpha}\left(\sum_{\alpha} \boldsymbol{K}_{\alpha \beta} \vec{\nabla} \mu^{\alpha}\right) \cdot \vec{n} d \Gamma, \\
\int_{\Omega} w_{\xi}\left(\frac{1}{J} \sum_{\beta} \frac{z^{\beta} F}{\bar{V}^{\beta}} \frac{D^{s} \Phi^{\beta}}{D t}\right) d \Omega=0 .
\end{array}\right.
$$

in which $\Gamma$ is the outer surface of the medium and $\boldsymbol{K}_{\alpha \beta}=\varphi^{\alpha} \varphi^{\beta}\left(\boldsymbol{B}^{\alpha \beta}\right)^{-1}$ a generalised diffusion-permeability tensor. We choose to use an updated Lagrange formulation. The total deformation tensor $\boldsymbol{F}$ may be divided into,

$$
\boldsymbol{F}=\boldsymbol{F}_{\Delta} \cdot \boldsymbol{F}_{n}
$$


where $\boldsymbol{F}_{n}$ describes the deformation from the initial configuration $\Omega_{0}$ to the reference configuration $\Omega_{n}$, and $\boldsymbol{F}_{\Delta}$ denotes the deformation from the reference configuration to the current state $\Omega$. When transforming the balance equations to a known domain, the reference configuration $\Omega_{n}$ is used. The gradient operator is transformed according to,

$$
\vec{\nabla}=\boldsymbol{F}_{\Delta}^{-c} \cdot \vec{\nabla}_{n}
$$

As the total deformation is divided, the volume ratio is divided in a similar way. From the definition of $J$ it follows that,

$$
J=J_{\Delta} J_{n}
$$

Time discretization of the material time derivatives for $J_{\Delta}$ and $\Phi^{\beta}$ yields,

$$
\begin{aligned}
J_{\Delta}\left(\vec{\nabla} \cdot \vec{v}^{s}\right) & =j_{\Delta}=\frac{J_{\Delta}-1}{\Delta t} \\
\frac{D^{s} \Phi^{\beta}}{D t} & =\frac{\Phi^{\beta}-\Phi_{n}^{\beta}}{\Delta t}
\end{aligned}
$$

For the mass balance a time discretization scheme is applied,

$$
\vec{\chi}=\theta \vec{\chi}\left(t_{n}+\Delta t\right)+(1-\theta) \vec{\chi}_{n}
$$

The time discretization scheme can be varied easily from implicit Euler $(\theta=1)$ to explicit Euler $(\theta=0)$. The Newton-Raphson iteration procedure is used to determine a sequence of approximate solutions of the non-linear equations. Quadratic interpolation functions $(\Phi)$ are used for the position field and weighing function $\vec{w}_{w}$. Linear interpolation functions $(\underset{\sim}{\Psi})$ are taken for the discretization of the pressure, electro-chemical potentials, electric potential, volume fractions and their corresponding weighing functions. The predictor is a set of linearized equations, 


\begin{tabular}{|c|c|c|c|c|c|c|}
\hline$\underline{S}$ & $-\underline{L}$ & $\underline{0}$ & $\underline{0}$ & $\delta \underset{\sim}{u}$ & & $-\underset{\sim}{R}$ \\
\hline$-\underline{L}^{T}$ & $-\sum_{\beta} \underline{C}_{\alpha \beta}$ & $\underline{C}_{\alpha \beta}$ & $-\sum_{\beta} \underline{C}_{\alpha \beta} \frac{z^{\alpha} F}{\bar{V}^{\alpha}}$ & $\delta p \underset{\sim}{p}$ & & $\underset{\sim}{U}-\sum_{\beta} \underset{\sim}{\underset{\beta}{Q}}$ \\
\hline$\underline{0}$ & $\underline{C}_{\alpha \beta}$ & $-\underline{K}_{\alpha \beta}-\underline{C}_{\alpha \beta}$ & $\underline{C}_{\alpha \beta} \frac{z^{\alpha} F}{\bar{V}^{\alpha}}$ & $\delta \mu_{\sim}^{\alpha}$ & & $\underset{\sim}{Q}+\sum_{\alpha}\left(\underset{\sim}{T}+\underset{\sim_{2}}{T}\right)$ \\
\hline$\underline{0}$ & $-\sum_{\beta} \underline{C}_{\alpha \beta} \frac{z^{\alpha} F}{\bar{V}^{\alpha}}$ & $\underline{C}_{\alpha \beta} \frac{z^{\alpha} F}{\bar{V}^{\alpha}}$ & $-\sum_{\beta} \frac{z^{\beta} F}{\bar{V}^{\beta}} \underline{C}_{\alpha \beta} \frac{z^{\alpha} F}{\bar{V}^{\alpha}}$ & $\delta \xi$ & & $-\sum_{\alpha} \frac{z^{\alpha} F}{\bar{V}^{\alpha}} \underset{\sim}{Q}$ \\
\hline
\end{tabular}

$$
\begin{aligned}
& \underline{S} \quad=\int_{\Omega} \underline{B}^{T}\left[\underline{D}_{F}+\underline{D}_{\tau}+\underline{D}_{J}\right] \underline{B} d \Omega \\
& \underline{L} \quad=\int_{\Omega} \underset{\sim}{B} \underset{w}{\Psi^{T}} d \Omega \\
& \underline{C}_{\alpha \beta}=\sum_{\alpha} \int_{\Omega} \underset{\sim}{\Psi} \frac{1}{J_{n} J_{\Delta}} C_{\alpha \beta} \Psi_{\sim}^{T} d \Omega \\
& \underline{K}_{\alpha \beta}=\theta \sum_{\alpha} \int_{\Omega} \underline{B}_{\mu}^{T} K_{\alpha \beta} \underline{B}_{\mu} \Delta t d \Omega \\
& \underset{\sim}{R}=\int_{\Omega} \underline{B}^{T} \underset{\sim}{\sigma} d \Omega \\
& \underset{\sim}{U}=\int_{\Omega} \Psi\left(\frac{J_{\Delta}-1}{J_{\Delta}}\right) d \Omega \\
& {\underset{\sim}{1}}_{1}=\theta \int_{\Omega} \underline{B}_{\mu}^{T} K_{\alpha \beta} \underline{B}_{\mu} \Delta t d \underset{\sim}{\mu^{\alpha}} \\
& \underset{\sim_{2}}{T}=(1-\theta) \int_{\Omega} \frac{1}{J_{\Delta}} \underline{B}_{\mu}^{T} \underline{F} K_{\alpha \beta} \underline{F}^{T} \underline{B}_{\mu} \Delta t d \underset{\sim_{\varphi}}{\alpha}
\end{aligned}
$$

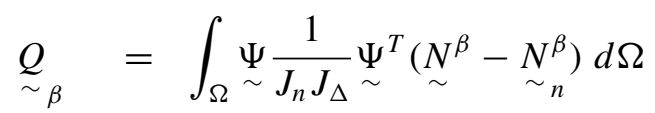


The matrices $\underline{D}_{F}, \underline{D}_{\tau}$ and $\underline{D}_{J}$ result from the linearisation of $\boldsymbol{F}_{\Delta}, \boldsymbol{\sigma}^{\text {eff }}$ and $J_{\Delta}$ respectively. The matrices $\underline{B}$ and $\underline{B}_{\mu}$ contain the derivatives of the quadratic and linear interpolation functions respectively. The column $B_{w}$ also contains of derivatives of the quadratic interpolation functions. For calculation a 27 -node brick element is chosen with 3 displacements in every node. In each corner of the brick one pressure, 3 chemical potentials and an electric potential is calculated, resulting in a total of 121 degrees of freedom per element. The code is verified using analytical solutions of the linearised equations for a 1D medium subject to stepwise change in external salt concentration [18]. The comparison is shown in fig. 1 for the electrochemical potentials of the cations and anions. The analytical solution is obtained by reducing the linearised equations to 3 diffusion equations. Both the numerical solution as the analytical solution solution clearly show two time constants, one for the diffusion of the ions and the other for the pressure diffusion.

\section{Discussion}

The present approach to porous media mechanics integrates, on the one hand, the rigourous mathematical framework of mixture theory and constitutive theory including equipresence, and on the other hand, the choice of Lagrangian independent and dependent variables as designed by Biot's physical intuitive insight into the correct variables that describe finite deformation of complex porous media. The result of this integration is an elegant and simple mathematical derivation of yet very generally applicable equations for finite deformation of ionised media. As a comparison,the reader should take time to compare the present formulation with those of $[6,14,5,11]$, and many others. The traditional Eulerian approach to the mechanics of mixtures leads to tedious mathematical derivations that results in many terms that are hard to interpret physically. Omong other complications, it leads to separate functions for the free energy of each constituent. Hence, the effort involved in evaluating these free energies is reduced by a factor $\mathrm{n}$ for a mixture of $n$ components, if a Lagrangian approach is used. The eq. (25) is a key result which justifies the choice of [3] to express the free energy $W$ as independent from the velocities (eq. 2.5 in his paper). Because, to our best knowledge, this independence from relative velocities cannot be shown for partial energies, 
the same assumption formulated for the partial free energies in [2] is far less obvious. So the experimental quantification of only one partial free energy is on itself more complicated than the quantification of the total energy $W$. The quadratic form of the total free energy (yielding linear constitutive relationships) for a 3D Lagrangian quadriphasic model has $\sum_{n=1,9} n=45$ parameters ( 6 strains, 3 volume fractions $=9$ ), whereas the corresponding form of the 4 Eulerian partial free energies has $4 \sum_{n=1,18} n=4 \cdot 171=684$ parameters ( 6 strains, 3 volume fractions, 3 times 3 velocity components $=18$ for each free energy). Lai et al. [11] have tried hard to introduce the electroneutrality restriction in an Eulerian form of the entropy inequality, and gave up because of the complexity of the resulting expressions (personal communication). [20] uses a partially Lagrangian formulation, which unfortunately is not sufficiently consistent to yield the advantage of a single energy function. Numerous authors, using an Eulerian formulation of mixture theory, gave up on the principle of equipresence because their equations became intractable $[6,1]$. These limitations disappear as a Lagrangian formulation is used. Finally, the transition to a Lagrangian description is rewarding for finding analytical and numerical solutions as well.

The specific choice of the form of the free energy (73) is the simplest of its kind that produces Donnan swelling. It is probably a rough approximation of the reality. It assumes that the mixing energy can be separated from the elastic energy. This separation is known in polymer science as the Flory-Rehner assumption, and has been disputed both for gels [19] as for biological tissue [10]. A more detailed description of the free energy of the mixture can be obtained along two tracks. One is the experimental route [12], the other is through micromechanics $[19,8,13]$. The micromechanics route can take advantage of the detailed knowledge available on electrostatic interactions. The best procedure is probably an integration of the experimental and micromechanical approach.

\section{Acknowledgement}

The authors acknowledge financial support from the Technology Foundation STW, the technological branch of the Netherlands Organisation of Scientific Research NWO and the ministry of Economic Affairs (grant MGN 3759). 


\section{REFERENCES}

[1] Achanta S., Cushman J. and Okos M., On multicomponent, multiphase thermodynamics with interfaces, Int. J. Eng. Sci. 32 (1984), 171-1738.

[2] Biot M.A., (1956). Theory of propagation of elastic waves in a fluid-saturated porous solid. i. low-frequency range, Journal of the Acoustical Society of America, 28 (1956), 168-178.

[3] Biot M.A., Theory of finite deformations of porous solids, Indiana University Mathematics Journal, 21 (7) (1972), 597-620.

[4] Biot M.A., Generalized lagrangian equations of non-linear reaction-diffusion, Chemical Physics, 66 (1982), 11-26.

[5] Boer R.d. and Ehlers W., Uplift, friction and capillarity: three fundamental effects for liquid saturated porous solids, Int. J. Solids Struct. 26 (1) (1990), 43-57.

[6] Bowen R.M., Incompressible porous media models by use of the theory of mixtures, Int. J. Engng Sci. 18 (1980), 1129-1148.

[7] Coleman B. and Noll W., The thermodynamics of elastic materials with heat conduction and viscosity, Archive of Rational Mechanics and Analysis, 13 (1963), 167-178.

[8] Galka A., Telega J.J. and Wojnar R., Modelling electric and elastic properties of cartilage, Engineering Transactions, 49 (2001), 283-313.

[9] Huyghe J.M. and Janssen J.D., Quadriphasic mechanics of swelling incompressible porous media, Int. J. Eng. Sci. 35 (1987), 793-802.

[10] Jin M.S. and Grodzinsky A.J., Effect of electrostatic interactions between glycosaminoglycans on the shear stiffness of cartilage, Macromolecules, 34 (2001), 8330-8339.

[11] Lai W.M., Hou J.S. and Mow V.C., A triphasic theory for the swelling and deformation behaviors of articular cartilage, J Biomech Eng. 113 (1991, Aug), 245-258.

[12] Lanir Y., Seybold J., Schneiderman R. and Huyghe J.M., Partition and diffusion of sodium and chloride ions in soft charged foam: the effect of external salt concentration and mechanical deformation, Tissue Engineering, 4 (4) (1998), 365-378.

[13] Moyne C. and Murad M., Electro-chemo-mechanical couplings in swelling clays derived from a micro-macro-homogenisation procedure, Int. J. Sol. Struct. 39 (2002), 6159-6190.

[14] Oomens C.W.J., van Campen D.H. and Grootenboer H.J., A mixture approach to the mechanics of skin, J. Biomechanics, 20 (9) (1987), 877-885.

[15] Richards E.G., An introduction to the physical properties of large molecules in solution (1 ed.). Cambridge: Cambridge University Press. ISBN: 0-521-23110-8 (1980).

[16] Staverman A., Non-equilibrium thermodynamics of membrane processes, Trans. Faraday Soc. 48 (1952), 176-185. 
[17] van Loon R., Huyghe J.M., Wijlaars M.W. and Baaijens F.P.T., 3d fe implementation of an incompressible quadriphasic mixture model, Int. J. Numer. Meth. Engng. 57 (2003), 1243-1258.

[18] van Meerveld J., Molenaar M.M., Huyghe J.M. and Baaijens F.T.P., Analytical solution of com pression, free swelling and electrical loading of saturated charged porous media, Transport in porous media, 50 (2003), 111-126.

[19] Vilgis T.A. and Wilder J., Polyelectrolyte networks: elasticity, swelling and the violation of the flory-rehner hypothesis, Comput. and Theor. Pol. Sci. 8 (1998), 61-73.

[20] Wilmanski K., Thermodynamic admissability of biot's model of poroelastic saturated materials, Arch. Mech. 54 (2002), 709-736. 